\title{
Online Feature Selection for Visual Tracking
}

Giorgio Roffo

giorgio.roffo@univr.it

Simone Melzi

simone.melzi@univr.it
Department of Computer Science

University of Verona

Verona, Italy
Object tracking is one of the most important tasks in many applications of computer vision. Many tracking methods use a fixed set of features ignoring that appearance of a target object may change drastically due to intrinsic and extrinsic factors. The ability to dynamically identify discriminative features would help in handling the appearance variability by improving tracking performance. The contribution of this work is threefold. Firstly, this paper presents a collection of several modern feature selection approaches selected among filter, embedded, and wrapper methods (e.g., Inf-FS [2], mRMR, SVM-RFE, among others). Secondly, we provide extensive tests, regarding the classification task, on the PASCAL VOC-2007 dataset intended to explore the strengths and weaknesses of the selected methods with the goal to identify the right candidates for online tracking. Taking advantage from the results obtained from the offline scenario, we decided to use the following four candidate methods: MutInf, Fisher, Inf-FS, and $m R M R$. In particular, we take care that execution times of these methods meet the requirements for a real-time application. Finally, we show how the selected algorithms can be successfully employed for ranking features used by the Adaptive Color Tracking (ACT) system proposed in [1], maintaining high frame rates. ACT system is one of the most recent solutions for tracking, it exploits color naming [3] (i.e., the action of assigning linguistic color labels to image pixels), to target objects and learn an adaptive correlation filter by mapping multi-channel features into a Gaussian kernel space. We evaluated four variants of ACT on the OTB-50 [4] benchmark, throughout three different robustness evaluation metrics: one-pass evaluation (OPE), temporal robustness evaluation (TRE), and spatial robustness evaluation (SRE) reporting the average precision and success rate for quantitative analysis. In Figure 1 only the top 10 (out of 34) trackers are displayed for clarity. In particular, the unsupervised method ACTinffs turns out to be the best trade-off between accuracy $(62.0 \%)$ and speed (111.4 fps). The ACTinffs has the

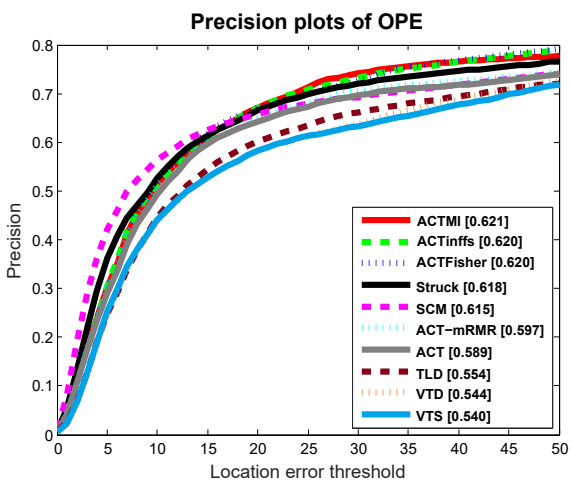

Figure 1: Precision plots over all 50 sequences provided by the OTB-50 benchmark. The mean precision scores for each tracker are reported in the legend.

same order of magnitude of the baseline ACT (196 fps) in terms of fps, while ACTMI operates at $19 \mathrm{fps}$. This work demonstrates the importance of feature selection for real-time applications, resulted in what is clearly a very impressive performance, our solutions improve up to $7 \%$ the baseline ACT while providing superior results compared to 29 state-of-the-art tracking methods.

[1] Martin Danelljan, Fahad Shahbaz Khan, Michael Felsberg, and Joost van de Weijer. Adaptive color attributes for real-time visual tracking. In IEEE Conf. Computer Vision and Pattern Recognition (CVPR), 2014.

[2] Giorgio Roffo, Simone Melzi, and Marco Cristani. Infinite feature selection. In IEEE Conf. International Conference on Computer Vision (ICCV), 2015.

[3] Joost van de Weijer, Cordelia Schmid, Jakob Verbeek, and Diane Larlus. Learning color names for real-world applications. IEEE Transactions on Image Processing, 2009.

[4] Yi Wu, Jongwoo Lim, and Ming-Hsuan Yang. Online object tracking: A benchmark. In IEEE Conference on Computer Vision and Pattern Recognition (CVPR), 2013. 